\title{
Correlation of phenolic content and antibacterial activity of dried lime extracts against human pathogens.
}

\author{
Dunia Alfarraj, Manal M Al Khulaifi, Nadine MS Moubayed* \\ Botany and Microbiology Department, Science College, King Saud University, Female Campus, Riyadh, Saudi Arabia
}

\begin{abstract}
Medicinal plants are the oldest known source for the treatment of diseases. The use of plants and plant extracts for isolation of pharmaceutical compounds has received increasing attention from researchers over the past decades. We determined the antibacterial activity of dried black lime (Citrus aurantifolia) extracts on Gram-positive and Gram-negative human pathogens, including Enterococcus faecalis, Staphylococcus aureus, methicillin-resistant Staphylococcus aureus (MRSA), Escherichia coli, Pseudomonas aeruginosa and Salmonella typhimurium by using agar well diffusion methods. Crude extracts were obtained from different organic solvents and from sterile distilled water as an aqueous solvent. Considerable antibacterial activity was observed for the methanolic extracts, particularly against $E$. faecalis. $P$. aeruginosa was the most susceptible among the Gram-negative bacteria. HPLC analysis of methanol dried lime extracts revealed a peak with a retention time of similar to that of the standard gallic acid, which is known to have potent antibacterial activity.
\end{abstract}

Keywords: Antibacterial, Dried lime extracts, HPLC, Gallic acid.

Abbreviations:

DLe: Dried Lime extracts; E. coli: Escherichia coli; E. faecalis: Enterococcus faecalis; MRSA: Methicillin-Resistant
Staphylococcus aureus; P. aeruginosa: Pseudomonas aeruginosa; S. aureus: Staphylococcus aureus; S. typhimurium: Salmonella typhimurium.

Accepted on August 29, 2018

\section{Introduction}

Plant-derived products have been used for medicinal purposes for many centuries. Herbs and spices, which are generally considered safe, have proven to be effective against certain ailments [1,2]. According to the World Health Organization, approximately $80 \%$ of the global population use herbs to treat ailments, with a lower usage in developed countries than in less developed countries [2]. As bacterial resistance to pharmaceutical agents becomes more common, several studies have focused on the antibacterial activities and potential use of plant-derived products, such as dried lime.

It is well-established that citrus fruits contain a wide range of bioactive compounds, such as phenolics, flavonoids, vitamins and essential oils, which are mainly responsible for the healthbeneficial effects, namely antimicrobial, anticancer, antiinflammatory, and antioxidant activities [3,4]. Lime (Citrus aurantifolia) is a shrub originally native to South East Asia and countries of the Middle East. The constituent flavonoids from the leaves, stem, roots, and flowers are known for their anticancer activity and antibacterial potential [5], antibacterial and antifungal properties [6,7] and anticancer and immunomodulatory effects in humans [8]. A previous study conducted on citrus fruits indicated that the Minimum Inhibitory Concentration (MIC) of lemon (C. lemon L.) peel was found to be 1:20 in the presence of methanol for Pseudomonas aeruginosa and MIC of 1:20 in the presence of acetone for Salmonella typhimurium [9]. Lemon and lime extracts were also found to inhibit the growth of vegetative cells and the spores of microorganisms, although the non-inhibitory concentrations of lemon extracts were found to be very low, at approximately $10 \mathrm{ppm}$ against Bacillus species and some lactic acid bacteria [10]. Another study confirmed the antibacterial activity of lemon against $S$. aureus, E. coli, and P. aeruginosa, with a mean zone of inhibition of $15-30 \mathrm{~mm}$, which was suggestive of the presence of flavonoids, tannins, alkaloids, glucosides, saponins, and terpenes that are believed to have potent therapeutic activity [10]. Gallic acid, a flavonoid has been validated through an HPLC analysis technique to have a retention time of $3.60 \mathrm{~min}$, which was in agreement with other pharmaceutical studies [11].

The present study was conducted to assess the antibacterial activity of aqueous and organic solvent extracts of the entire fruit of dried lime against six bacterial species. The presence of gallic acid, to which this antibacterial activity was attributed, was revealed through HPLC analysis and comparison with a gallic acid standard. 


\section{Methods}

\section{Preparation of dried lime extracts (DLE)}

Pre-dried lime pieces, dried at room temperature, were purchased from a local market in Riyadh, Saudi Arabia. The dried lime fruit samples were ground into a powder and $10 \mathrm{~g}$ was extracted with $100 \mathrm{ml}$ of methanol, acetone, and ethanol, and water. The samples were incubated for $3 \mathrm{~d}$ at $27^{\circ} \mathrm{C}$ in a rotating shaker at $120 \mathrm{rpm}$ (Innova Lumberick, Thomas Scientific, Germany), filtered through Whatman (number 2) filter paper and aseptically filtered through a $0.45 \mu \mathrm{m}$ filter (Millipore, Merck, USA) [10]. The filter-sterilized DLEs were stored in sterile Eppendorf tubes at $-4^{\circ} \mathrm{C}$ until use.

\section{Bacterial suspension preparation}

The pure cultures of the standard microbial isolates tested in this study were obtained from King Khalid University Hospital, Microbiology Laboratory, Riyadh, Saudi Arabia. Bacterial isolates, including Gram-positive bacteria such as Staphylococcus aureus (ATCC 25923), methicillin-resistant Staphylococcus aureus (MRSA ATCC 12498) and Enterococcus faecalis (ATCC 29212) and Gram-negative bacteria, namely Escherichia coli (ATCC 25966), Pseudomonas aeruginosa (ATCC 27853) and Salmonella typhimurium (LT2), were grown on nutrient agar plates and nutrient broth (Oxoid). Small inoculums of the microbial isolates were suspended in $5 \mathrm{ml}$ sterile nutrient broth to prepare microbial suspensions of 0.5 MacFarland turbidity.

\section{In-vitro antibacterial assay}

Antibacterial activity was assayed by using the agar well diffusion technique described by Das et al. [12]. This activity was evaluated through the measurement of the inhibition zone around each well loaded with the extract. Each of the microbial suspensions prepared was loaded on sterile Muller Hinton agar plates surface (Oxoid, UK) by using sterile cotton swabs. The loaded plates were then perforated at equal intervals with a sterile cork borer (diameter, $6 \mathrm{~mm}$ ). Seventy microliters of each of the DLEs (acetone, methanol, ethanol, and water) was loaded respectively into the appropriate wells. The plates were left to stand for $30 \mathrm{~min}$ at room temperature and then incubated at $37^{\circ} \mathrm{C}$ for $18-24 \mathrm{~h}$.
Antimicrobial activity was evaluated through the measurement of the inhibition zone. All tests were performed in duplicate and the mean size of the inhibition zone was recorded in millimeters. Standard antibiotic discs (Oxoid, UK) of ampicillin $(10 \mu \mathrm{g})$, vancomycin $(30 \mu \mathrm{g})$, trimethoprimsulfamethoxazole $(25 \mu \mathrm{g})$, and tetracycline $(30 \mu \mathrm{g})$ were used as the positive controls and comparative agents for the study of the efficacy of the DLEs.

\section{HPLC analysis}

Analytical High-Performance Liquid Chromatography (HPLC, Shimadzu, Japan) was used to determine the predominant chemical constituents of the methanol DLE, compared with a standard gallic acid (GA), by separation on a $\mathrm{C}_{18}$ column at $25^{\circ} \mathrm{C}$. Briefly, $30 \mu \mathrm{L}$ of GA and methanol DLE were each filtered through $0.45 \mu \mathrm{m}$ syringe filters (Millipore, USA). The filtrate was then injected onto the column for a total run time of $45 \mathrm{~min}$, with UV detection at $254 \mathrm{~nm}$. The mobile phase was water: methanol: tetrachloroacetic acid in the following ratio (90:1:1).

All results were analysed statistically by using the Predictive Analysis Software (PASW) version 20 (SPSS Inc., IBM, Chicago, Illinois, USA).

\section{Results}

The comparison of the antibacterial activity of DLE with that of standard commercial antibiotic discs is shown in Table 1. The average inhibition zone of the methanol DLE was the largest against $E$. faecalis $(28 \mathrm{~mm})$, followed by $S$. aureus and MRSA, for which the average inhibition zone was $21 \mathrm{~mm}$ (Figure 1A). All DLEs used for E. coli resulted in zones of inhibition of between 15 and $16 \mathrm{~mm}$ (Figures 1B-1D). The methanol and acetone DLEs used against $P$. aeruginosa resulted in zones of inhibitions of $26 \mathrm{~mm}$ and $25 \mathrm{~mm}$, respectively (Figure 1E). Similarly, the methanol DLE used for S. typhimurium resulted in an $18 \mathrm{~mm}$ zone of inhibition (Figure $1 \mathrm{~F})$. These data were comparable with that obtained for the standard antibiotic discs, in which the inhibition zones varied between $22 \mathrm{~mm}$ and $28 \mathrm{~mm}$, which indicated the potent antibacterial activity of the extracts.

Table 1. Average inhibition zones of DLEs (in mm) against Gram-positive and Gram-negative human pathogens.

\begin{tabular}{|c|c|c|c|c|c|c|}
\hline \multicolumn{7}{|c|}{ Average zone of inhibition } \\
\hline \multirow[t]{2}{*}{ Organisms } & \multicolumn{4}{|c|}{ Solvent used for DLE production } & \multicolumn{2}{|l|}{ Positive control } \\
\hline & Methanol & Acetone & Ethanol & Water & Antibiotic $(\mu \mathrm{g})$ & Zone of inhibition \\
\hline Staphylococcus aureus & $21 \mathrm{~mm}$ & $19 \mathrm{~mm}$ & $18.5 \mathrm{~mm}$ & $19 \mathrm{~mm}$ & Ampicillin $10 \mu \mathrm{g}$ & $29 \mathrm{~mm}$ \\
\hline Enterococcus faecalis & $28 \mathrm{~mm}$ & $24 \mathrm{~mm}$ & $25.5 \mathrm{~mm}$ & $23 \mathrm{~mm}$ & SXT $25 \mu \mathrm{g}$ & $27 \mathrm{~mm}$ \\
\hline MRSA & $21 \mathrm{~mm}$ & $18 \mathrm{~mm}$ & $19 \mathrm{~mm}$ & $15.5 \mathrm{~mm}$ & Vancomycin $30 \mu \mathrm{g}$ & $25 \mathrm{~mm}$ \\
\hline Escherichia coli & $16 \mathrm{~mm}$ & $15 \mathrm{~mm}$ & $15 \mathrm{~mm}$ & $15 \mathrm{~mm}$ & Ampicillin $10 \mu \mathrm{g}$ & None \\
\hline
\end{tabular}




\begin{tabular}{llcllll}
\hline Pseudomonas aeruginosa & $26 \mathrm{~mm}$ & $25 \mathrm{~mm}$ & $23 \mathrm{~mm}$ & $22 \mathrm{~mm}$ & Tetracycline $30 \mu \mathrm{g}$ \\
\hline Salmonella typhimurium LT2 & $18 \mathrm{~mm}$ & $15 \mathrm{~mm}$ & $16 \mathrm{~mm}$ & $15 \mathrm{~mm}$ & SXT 25 $27 \mathrm{mg}$ & $19 \mathrm{~mm}$ \\
\hline
\end{tabular}

Note: The diameter of the zone of inhibition $(\mathrm{mm})$ is presented as the mean of three replicates. SXT: Trimethoprim-Sulfamethoxazole.

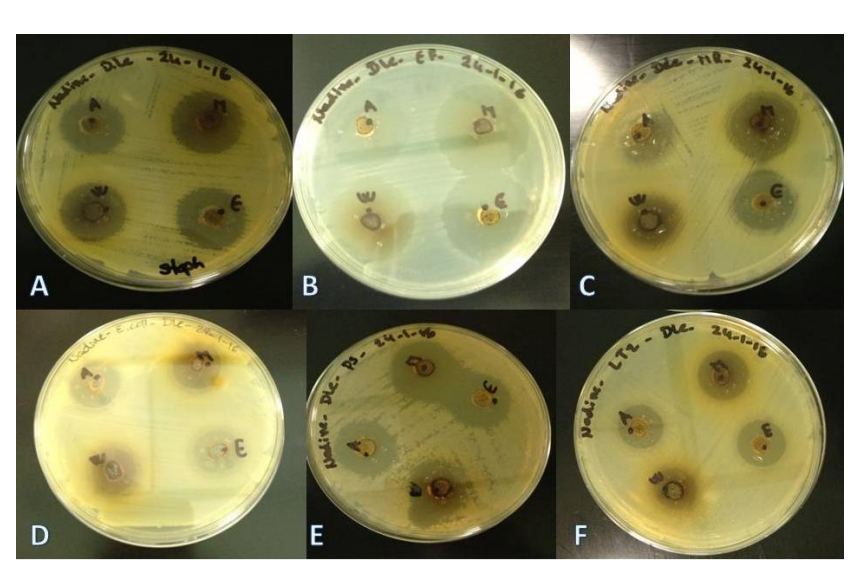

Figure 1. A-F. Zones of inhibition (in $\mathrm{mm}$ ) of DLEs produced using four different solvents: methanol, acetone, water and ethanol against $S$. aureus (A), E. faecalis (B), MRSA (C), E. coli (D), P. aeruginosa (E), and S. typhimurium (F). A: Acetone; DLE M: Methanol DLE; E: Ethanol DLE extract W: Water DLE.

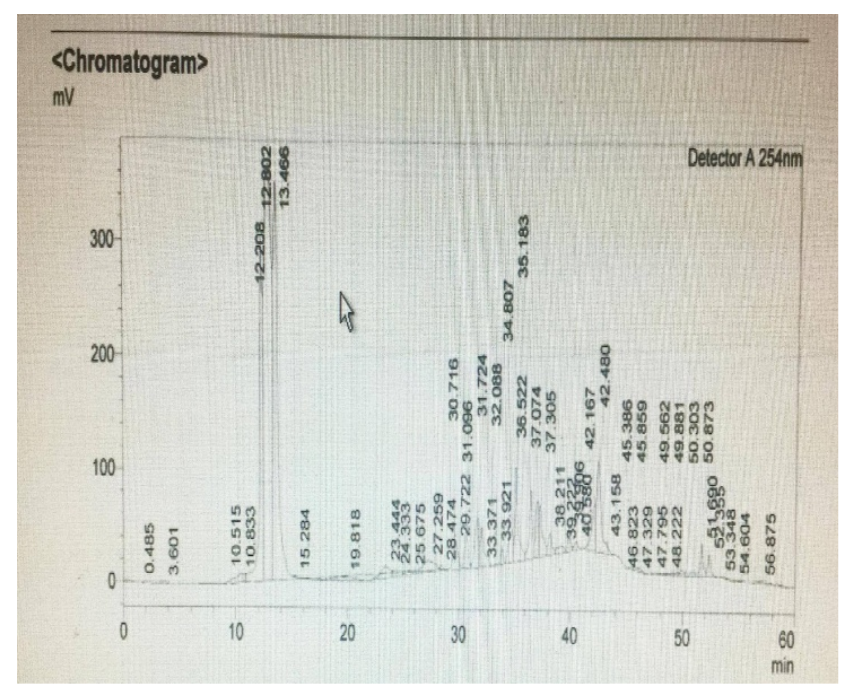

(a)

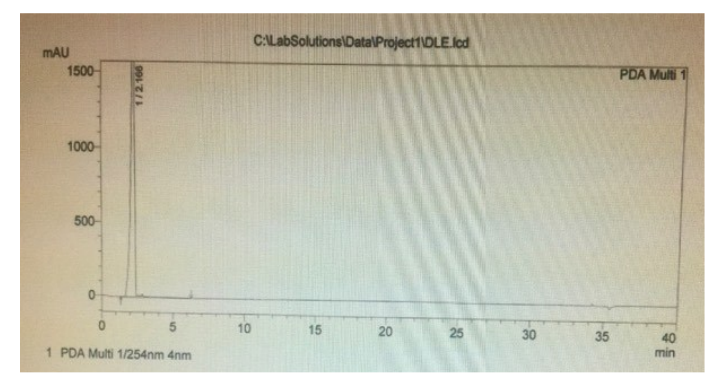

(b)

Figure 2. HPLC chromatogram for methanol DLE (a) and the gallic acid standard (b).

The HPLC analysis of methanol DLE revealed a peak with a retention time of 12.2 corresponding to the presence of gallic acid; the peak of the standard gallic acid had a retention time of 3.6 this difference in retention time was due to volume injected, the HPLC device used and the running time (Figure 2).

\section{Discussion}

Over the past few decades, the antibacterial potential of plantderived extracts has been studied extensively owing to the emergence and increased resistance of several microorganisms to pharmaceutical agents $[1,2]$.

This study has focused on the antibacterial properties of lime fruit extracts in their dried state in line with the reports of Burt [6] and Ortuno et al. [7] which showed that lime contains flavonoids with both antibacterial and antifungal properties. In contrast with their studies, this study demonstrated the antibacterial activity against $S$. aureus, E. faecalis, E. coli, MRSA, $P$. aeruginosa and $S$. typhimurium (Table 1). Furthermore, in this study, the antibacterial activity of methanol DLE, as indicated by a zone of inhibition against $S$. aureus of $21 \mathrm{~mm}$, was almost comparable with the $29 \mathrm{~mm}$ zone of inhibition produced by the standard antibiotic ampicillin against $S$. aureus. The same was true for the comparison of the zone of inhibition produced by methanol DLE with the antibacterial effect of vancomycin on MRSA (21 mm with methanol DLE vs. $25 \mathrm{~mm}$ with vancomycin). In contrast, the antibacterial effects of methanol DLE against $E$. faecalis (28 $\mathrm{mm}), P$. aeruginosa $(26 \mathrm{~mm})$ and $S$. typhimurium $(18 \mathrm{~mm})$ were comparable, if not higher than, the zones of inhibition produced by sulfamethoxazole $(27 \mathrm{~mm})$, tetracycline $(27 \mathrm{~mm})$, and sulfamethoxazole $(19 \mathrm{~mm})$, respectively.

These results confirmed the findings of Dhanavade et al. [9] with respect to the antibacterial activity of lemon (peel) against $P$. aeruginosa, and $S$. typhimurium. The HPLC results of this study confirmed the presence of gallic acid in the methanol DLE (Figure 2), which may explain the antibacterial activity of the DLEs. Gallic acid, which is abundant in plants and fruits, is reported to be active against $S$. aureus [13]. Furthermore, gallic acid, alone or in combination with tannic acid, was found to impair bacterial growth levels of Chromobacterium [14]. Other researchers showed that gallic acid, in combination with ampicillin and penicillin, had potent antibacterial activity against MRSA [15].

The results obtained in this study indicated that the antibacterial activity of DLEs was highly correlated with the presence of phenolic compounds, particularly gallic acid. 


\section{Conclusion}

The DLEs, in particular the methanolic extract, exerted significant antibacterial activity against Gram-positive and Gram-negative human pathogens, including $S$. aureus, E. faecalis, E. coli, MRSA, P. aeruginosa and S. typhimurium. The DLEs were found to contain gallic acid, which may be the cause of their antibacterial activity and may represent a promising lead for the development of a clinical treatment.

\section{Acknowledgement}

This research project was supported by a grant from the "Research Center of the Center for Female Scientific and Medical Colleges", Deanship of Scientific Research, King Saud University. The authors thank the Deanship of Scientific Research and RSSU at King Saud University for their technical support.

\section{References}

1. Shelef LA. Antimicrobial effects of spices. J Food Safety 1983; 6: 29-44.

2. Zaika LL Spices and herbs: their antimicrobial activity and its determination. J Food Safety 1988; 9: 97-118.

3. Aruoma OI, Landes B, Ramful-Baboolall D, Bourdon E, Neergheen-Bhujun V, Wagner KH, Bahorun T. Functional benefits of citrus fruits in the management of diabetes. Prev Med 2012; 54 Suppl: S12-16.

4. Karimi E, Oskoueian E, Hendra R, Oskoueian A, Jaafar HZE. Phenolic compounds characterization and biological activities of Citrus aurantium bloom. Molecules 2012; 17 : 1203-1218.

5. Mohanapriya M, Ramaswamy L, Rajebdran R. Health and medicinal properties of lemon (Citrus limolium). Int $\mathrm{J}$ Ayurvedic Herbal Med 2013; 3: 1095-1100.

6. Burt S. Essential oils: their antibacterial properties and potential applications in foods-a review. Int $\mathrm{J}$ Food Microbiol 2004; 94: 223-253.

7. Ortuno AA, Baidez P, Gomez MC, Arcas I, Porras AG, Del Rio JA. Citrus paradise and Citrus sinensis flavonoids: their influence in the defense mechanism against Penicillium digitatum. Food Chem 2006; 98: 351-358.

8. Arias BA, Ramon-Laca L. Pharmacological properties of citrus and their ancient and medieval uses in the Mediterranean region. J Ethnopharmacol 2005; 97: 89-95.
9. Dhanavade MJ, Jalkute CB, Ghosh JS, Sonawane KD. Study antimicrobial activity of lemon (Citrus lemon L.) peel extract. Br J Pharm Toxicol 2011; 2: 119-122.

10. Penecilla GL, Magno CP. Antibacterial activity of extracts of twelve common medicinal plants from the Philippines. J Medicinal Plants 2011; 5: 3975-3981.

11. Kardani K, Gurav N, Solanki B, Patel P, Patel B. RPHPLC method development and validation of gallic acid polyherbal tablet formation. J Appl Pharm Sci 2013; 3: 37-42.

12. Das K, Tiwari RKD, Shrivastava DK. Techniques for evaluation of medicinal plant products as antimicrobial agent: current methods and future trends. J Medic Plant Res 2010; 4: 104-107.

13. Lima VN, Oliveira-Tintino CD, Santos ES, Morais LP, Tintino SR, Freitas TS, Geraldo YS, Pereira RL, Cruz RP, Menezes IR, Coutinho HD. Antimicrobial and enhancement of the antibiotic activity by phenolic compounds: gallic acid, caffeic acid and pyrogallol. Microb Pathog 2016; 99: 56-61.

14. Dusane DH, OMay C, Tufenkji N. Effect of tannic and gallic acids alone or in combination with carbenicillin or tetracycline on Chromobacterium violaceum CV026 growth, motility and biofilm formation. Can J Microbiol 2015; 6: 487-494.

15. Lee DS, Eom SH, Kim YM, Kim HS, Yim MJ, Lee SH, Lee $\mathrm{SH}$, Kim DH, Je DJY. Antibacterial and synergic effects of gallic acid-grafted-chitosan with $\beta$-lactams against methicillin-resistant Staphylococcus aureus (MRSA). Can J Microbiol 2014; 60: 629-638.

\section{*Correspondence to}

Nadine MS Moubayed

Microbiology Department

Science College

King Saud University

Female Campus

Saudi Arabia

Email: nmoubayed@ksu.edu.sa 